\title{
Computed tomography scans of the thorax
}

\section{La tomographie du thorax}

$\mathrm{I}$ $\mathrm{n}$ the present issue of the Canadian Respiratory Journal, Turner et al (pages 311-316) describe a prospective study of thoracic computed tomography (CT) scanning at Vancouver General Hospital (Vancouver, British Columbia). They essentially described the hospital's workload of thoracic CT scans, and used a pre- and postscan questionnaire to assess the diagnostic impact of the scan findings and the appropriateness of the timing of the scan according to priorities that ranged from 'elective' to 'emergency'. They were able to collect usable data - pre- and postscan questionnaire results - from $62 \%$ of the patients examined, amounting to 454 cases. This is a substantial number, which I interpret as indicating a high level of cooperation between the radiologists and the referring physicians who filled out the questionnaires.

Many of their results were predictable, I think, given the environment. Most scans were ordered by respirologists, with thoracic surgeons coming in second. Most, especially those carried out in outpatients, were for diagnostic purposes as opposed to observing the progress of a known disease. Surgeons were more likely to order scans for staging, and respirologists for diagnosis. Indications and wait times differed substantially between inpatients and outpatients.

Impacts were assessed in terms of whether the initial diagnosis was changed by the scan result; in $52 \%$ of cases, this did not occur, while in $48 \%$, it did. One-quarter of the scans were normal and, in 23\%, the diagnosis was changed from one disease to another. In $56 \%$ of cases, referring physicians indicated that the results of the scan generated important new information. Although I am sure that these results are accurate and reflect the major contribution that CT scans make to the current diagnosis of lung diseases (see below), I believe the 'change versus nonchange' dichotomy is an oversimplified view of clinical decision-making. No one is going to send in a CT requisition with a working diagnosis of 'normal', although I am sure that many were ordered to see if they were in fact normal. A particularly good example of this is the suspicion of pulmonary embolism: $68 \%$ of the scans investigating this disease were normal, and I am sure that many were performed precisely to ascertain if this was the case. The case is similar for many of the scans for nodules and interstitial lung diseases, other major contributors to the normal category. It would also be somewhat surprising if in the cases in which the disease category was changed, the new diagnosis had not been considered by the referring physician. Turner et al tried to get a feel for this kind of situation by asking about the confidence of the referring physician in the initial and subsequent diagnoses, but this is a tricky business, and results were not very striking. The largest increase in confidence occurred when scans were normal, as may have been expected given the sensitivity of the technique. ans le présent numéro du Canadian Respiratory Journal, Turner et coll. (pages 311-316) résument une étude prospective sur les tomographies du thorax réalisées au Vancouver General Hospital (Vancouver, Colombie-Britannique). Les auteurs ont essentiellement fait un bilan des tomographies du thorax réalisées dans cet établissement et ils ont utilisé des questionnaires pré- et post-test pour évaluer l'impact du diagnostic sur l'analyse des résultats et sur les temps d'attente en fonction de demandes allant de «électives » à « urgentes ». Ils ont ensuite compilé les résultats des questionnaires, qui se sont révélés utilisables pour $62 \%$ des patients examinés, c'est-à-dire 454 cas. Il s'agit d'une proportion substantielle que j'interprète comme le signe d'une excellente coopération entre les radiologues et les médecins demandeurs qui ont accepté de répondre aux questionnaires.

Plusieurs conclusions m'ont semblé prévisibles, compte tenu du contexte. La plupart des tomographies ont été demandées par des pneumologues, les chirurgiens thoraciques venant au second rang parmi les demandeurs. La majorité des ces examens, et particulièrement ceux qui étaient effectués en externe, étaient demandés pour des raisons diagnostiques, par opposition à des examens pour le suivi d'une maladie connue. Les chirurgiens avaient davantage tendance à demander une tomographie pour stadifier un état pathologique et les pneumologues, pour le diagnostiquer. Les indications et les temps d'attente différaient substantiellement selon que les patients étaient hospitalisés ou non.

Les impacts ont été mesurés selon que les résultats de la tomographie modifiaient le diagnostic initial; dans $52 \%$ des cas, le diagnostic n'a pas changé. Un quart des tomographies se sont révélées normales et $23 \%$ ont donné lieu à un changement de diagnostic. Dans 56 \% des cas, les médecins demandeurs ont inscrit que les résultats de la tomographie avaient révélé d'importants nouveaux indices. Je suis persuadé que ces résultats sont exacts et ils illustrent bien l'importance de la tomographie aujourd'hui dans le diagnostic des maladies pulmonaires (voir plus loin), mais il me semble que cette dichotomie " changement versus absence de changement » simplifie à outrance le processus décisionnel clinique. Personne ne demandera une tomographie si le diagnostic provisoire est « sans particularité », même si je suis persuadé que bon nombre d'examens visent précisément à confirmer que l'état du patient est normal. Un exemple particulièrement éloquent de ce phénomène est le soupçon d'embolie pulmonaire : $68 \%$ des tomographies effectuées pour confirmer cette entité clinique se sont révélées normales et je suis persuadé que plusieurs ont été effectuées précisément pour confirmer que tel était le cas. La situation est similaire pour beaucoup de tomographies demandées chez des patients présentant une maladie pulmonaire interstitielle ou des nodules, autres diagnostics que la 
I should note that the above criticisms are relatively easy to make, but it would be extremely difficult to design a study that answered them in a comprehensive way in a large series of cases.

Of equal interest was the analysis of the time between receipt of the requisition for the CT scan and its execution. Elective cases had a mean wait time of approximately 30 days, while moderate and urgent cases waited less than one week. The wait time for suspected cancer was 12 to 14 days. The referring physician prioritized individual patients, and the results indicate a high degree of concurrence on the part of the radiologists with or without informal communication. In only $4 \%$ of cases did the referring physicians feel that the scan was overly delayed. The authors characterize this as reflecting the Canadian health care system. I hope this is the case.

Finally, I must comment that thoracic CT scans are a major advance in the diagnosis of lung disease. They give fantastic anatomical detail and because of this are (I think) easier to read than chest $\mathrm{x}$-rays. They are absolutely essential in the diagnosis and management of lung cancer, interstitial disease and pulmonary embolism, at least. Presumably because of this, they have become much easier to obtain as the number of available scanners has grown; I have no problem in getting prompt outpatient scans in my patients with interstitial disease in Manitoba, and that is a good thing.

Nick R Anthonisen MD Editor-in-Chief, Canadian Respiratory Journal tomographie permet d'écarter. Il serait en outre quelque peu étonnant que, le cas échéant, le nouveau diagnostic établi au moyen de la tomographie n'ait jamais effleuré l'esprit du médecin demandeur. Turner et ses collègues ont essayé d'analyser la situation sous cet angle, en vérifiant le degré de confiance des médecins demandeurs à l'endroit des diagnostics initiaux et subséquents. Or, la question était délicate et les résultats n'ont rien révélé d'étonnant. La confiance la plus marquée se manifestait à l'endroit des tomographies normales, ce qui est prévisible compte tenu de la sensibilité de cette technique. Admettons toutefois qu'il est facile de formuler des critiques, alors que dans les faits, ce serait un véritable tour de force que d'arriver à concevoir une étude capable de répondre à la question globalement et pour un grand nombre de cas.

L'analyse du délai entre la réception de la demande de tomographie et sa réalisation s'est révélée tout aussi intéressante. Dans les cas électifs, les temps d'attente ont été d'environ 30 jours, tandis que dans les cas modérés et urgents l'attente a duré moins d'une semaine. Le temps d'attente pour un cancer soupçonné, par exemple, était de 12 à 14 jours. Les médecins demandeurs priorisaient leurs patients sur une base individuelle et les résultats indiquent un fort degré de collaboration avec les radiologues, avec ou sans communication informelle. Les médecins demandeurs ont estimé que la durée de l'attente avait été excessive dans $4 \%$ seulement des cas. Les auteurs jugent que c'est un reflet fidèle de la situation des soins de santé au Canada. J'espère qu'ils disent vrai.

En terminant, précisons que la tomographie du thorax constitue une percée majeure dans le diagnostic de la maladie pulmonaire. Les tomographies révèlent des détails anatomiques fantastiques. C'est pourquoi, à mon sens, elles sont plus faciles à interpréter que les radiographies. Elles sont absolument indispensables au diagnostic et à la prise en charge du cancer du poumon, de la maladie interstitielle et de l'embolie pulmonaire pour ne nommer qu'eux. C'est probablement pourquoi, elles sont devenues beaucoup plus accessibles à mesure que les appareils se sont multipliés dans les établissements de santé. Pour ma part, je n'ai jamais de difficulté à obtenir des tomographies pour mes patients manitobains non hospitalisés s'ils souffrent de maladie interstitielle et c'est très bien ainsi. 


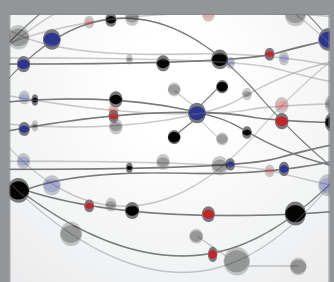

The Scientific World Journal
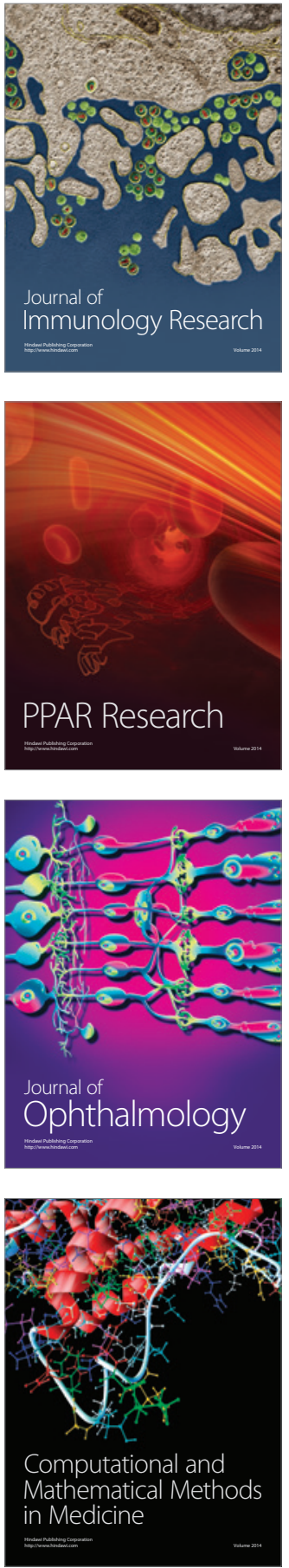

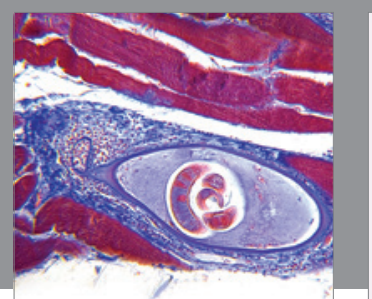

Gastroenterology Research and Practice

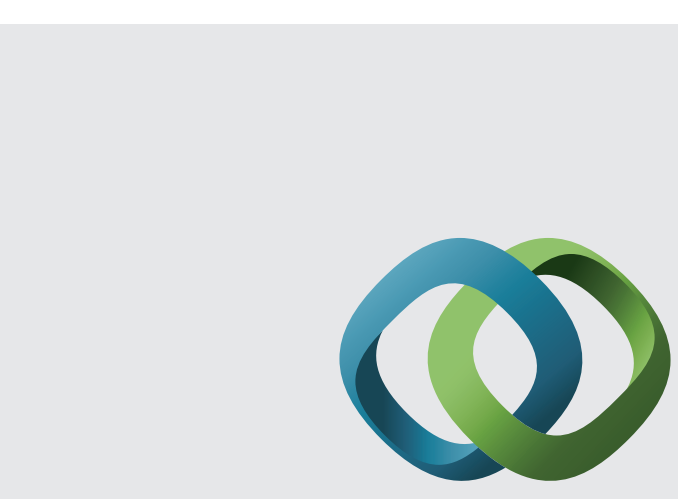

\section{Hindawi}

Submit your manuscripts at

http://www.hindawi.com
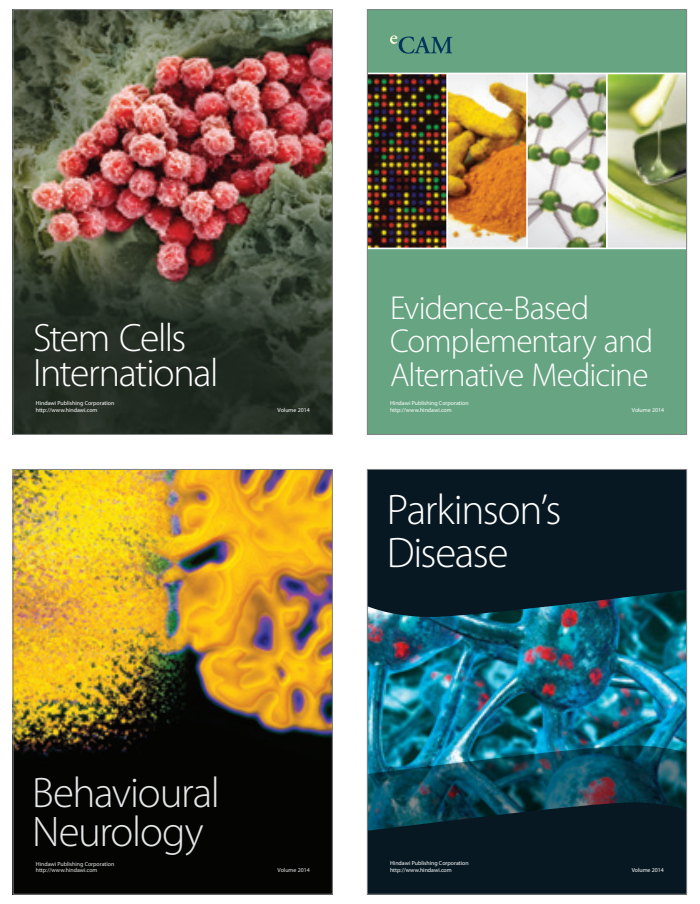
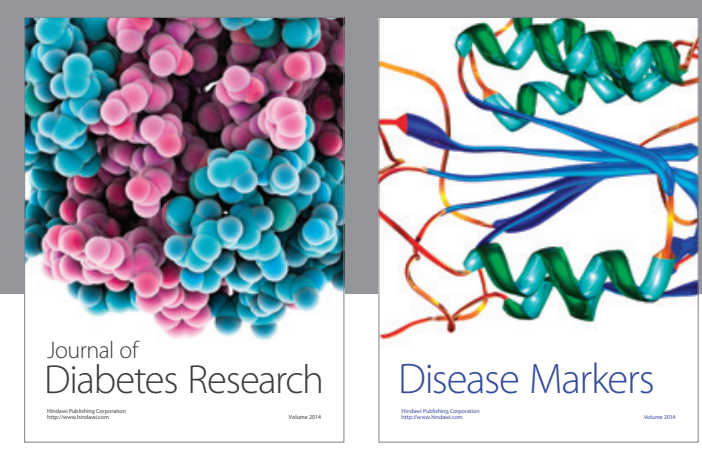

Disease Markers
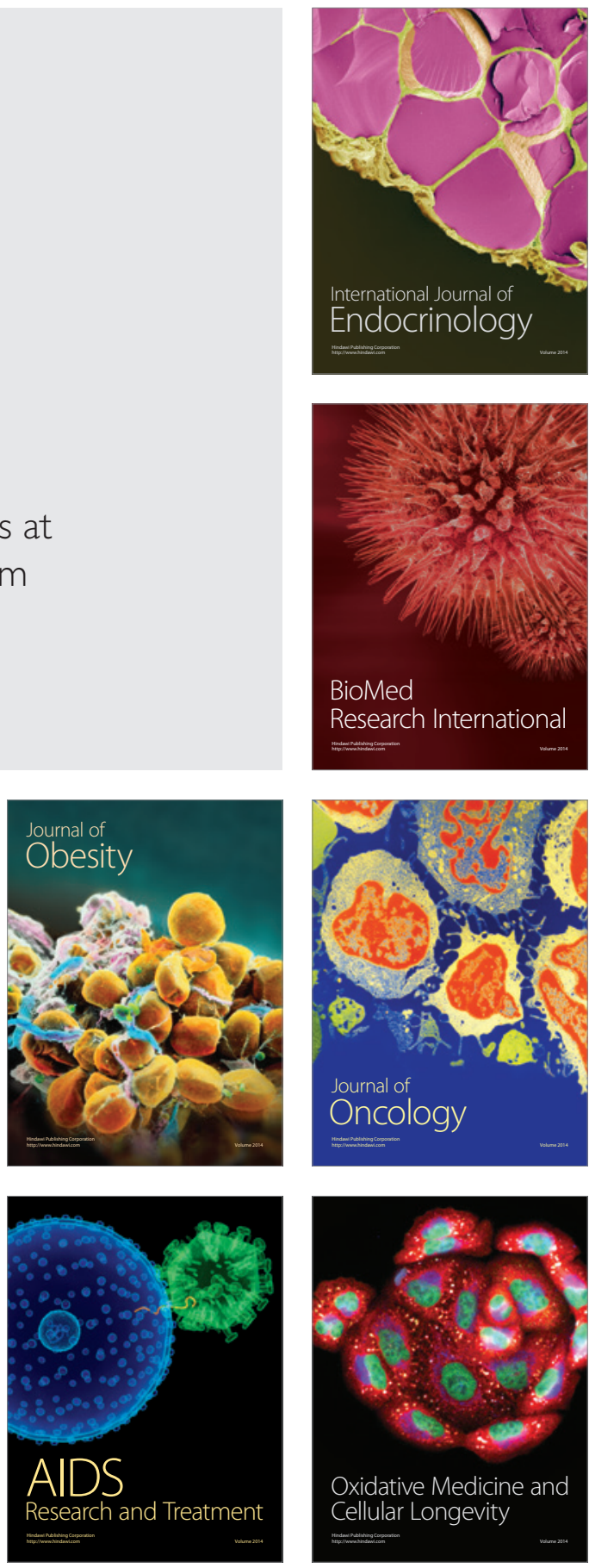